\title{
Simulation Study of Aerodynamics during the Tennis Flight- A Case Study about the Topspin
}

\author{
Zhang Qing $^{1, a}$, Zhang Chunyan ${ }^{1, b}$, Zhang Qiang ${ }^{2, c}$
}

1. School of Sports, China University of Mining and Technology, Xuzhou 221116, China;

2. School of Safety Engineering, China University of Mining and Technology, Xuzhou 221116, China

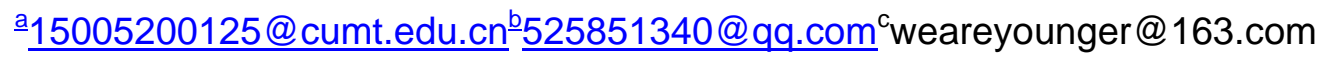

Keywords: Computational fluid dynamics, topspin, mechanics, aerodynamics

\begin{abstract}
The pressure distribution of airflow field around tennis and the characteristics of gas under various rotational velocities and flight conditions of tennis are simulated by the method of computational fluid dynamics(CFD) andtherelational software fluent 14.5 is used. Then the pressure distribution on the upper and lower surface of tennis are analyzed point by point. The results indicate thatdifference in pressure between the upper and lower surface of tennis increases with the increase of rotational speed,which can enhance the effect of Magnus force and causethe rapid falling of tennis. The increase of tennis speed will disorder the flow of the surrounding gas, result in the worse motion movement controllability oftennis, and change the motion trajectory. The static pressure distribution on the upper and lower surfaces oftennis is similar, but the change of pressure for the lower surface is slightly behind that of the upper surface. The drag coefficient and lift coefficient increase with the increase of the tennis speed and the rotational velocity, respectively.
\end{abstract}

\section{Introduction}

The complex mechanical principle exists in the flight process of tennis, which includes many kinds of forces with synergistic continuous action. In this case, tennis always rotates following the specific direction.Forehand, as the most powerful weapon of tennis players, not only enhance the ball stability, but also improvestrongly bounced back height, which canincreasethe difficulty in return [1]. The most representative is that Rafael Nadal returns tennis with more than 3000rpm in clay court of theFrench Open, whichmakes Federer, Murray, Djokovic and many other players difficult to havethe high-quality counterattacks. The rotationalball drivesthe surrounding air, andthe airflow rate of the spherical side is greater than the other side. According to the Bernoulli equation, the pressure for the position with the greater velocity is relatively small, which can produce a force perpendicularly to the topspin flight direction[2]. Under the effect of this force, the opponent is difficult to predict andit is not easy to returntennis due to the extended flight time, decreased arc over net, and higher rebound after landing.Therefore, it is of great significance to study the characteristics offlying process for topspintennis.

For the high-speed flyingtennis, the research on the topspin mainly bases on observing video of excellent players with different skills, but it is difficult to track the aerodynamics characteristics of the flight process only by qualitative analyses. In this paper, the aerodynamic characteristics of the different spherical positions of tennis in the flight process are simulated and analyzed. And the surrounding airflow field and its dynamic parameters during the flight process of atennis are studied by the method of computational fluid dynamics. This research work provides a reference for improving the tennis technical, skills tactical, daily training and competition.

\section{Mechanical factors of the flight tennis}

A tennis during the flight process may be subject to various forces, such as gravity, airflow resistance, Magnus force caused by rotation, the virtual mass force and Bassett force caused by the relative speed between tennis and air, etc. The main factors that affect the trajectoryof tennis are 
gravity, air resistance, Magnus force, and so on[3,4].The air resistance has the greatest impact on the flight trajectory of tennis.Many factors can affect the air resistance, including the Reynolds number of the flow field around the tennis, the compressibility of airflow, the turbulence characteristics, and the roughness of tennis surface[5-7]. The topspin tennis rotates along the corresponding direction because of the certain torque. This may cause the viscous role of the entrainment flow aroundtennis.This process affectsthe air velocity distribution in different sides oftennis,and causes the difference in pressure for two opposite directions. This is the Magnus effect in rotation. The non uniform motion oftennis not only changesthe tennis speed, but also drives the surrounding airflow changing in the same way, which is called virtual mass force. Iftennis is assumed as a standard sphere, its virtual mass force is equal to half of buoyancy force. The magnitude of this force is proportional to the difference between the acceleration of tennis and airflow. The high-speed ball will drive the air onsurface, and the air on the surface will inhibit the changes in the trend of tennis speed. This effect is known as the Bassett force. The changes of the forcerelate to the speed changes of tennis. If the speed changes violently, the resistance is much higher than that in steady state. The physical meaning of Bassett force is the time integral of additional viscous action, which is the movement of tennis relative to air.

This article aims to simulatethe characteristics of airflow field around tennis in various movements. The buoyancy, virtual mass force and Bassett forceare small in the movement of tennis, and their impacts on airflow field characteristics and the kinetic parameters of tennis movement are negligible. Therefore, the gravity, drag and Magnus force are added to the momentum conservation equation in the simulation. Thedistribution of airflow field on the upper and lower surfaceof tennis varies with the flight speed and direction. In this simulation, the effects of flight speed and rotational speed on the characteristics of airflow field around the tennis, the pressure distribution and the aerodynamic parameters are investigated with the example of topspin. Simulation tests of two factors and multi-levelsare conducted.

\section{Simulation of the flight process of tennis}

3.1 Meshing.Considering the complexity and importance of the flow field on the surface of atennis, a boundary layer mesh is used to partition the surface to achieve the mesh encryption. The method of partitioning meshes is adopted in the other zone of flow field. The mesh of the flow field around the spherical surface is a method of non-structural meshing with quadrilateral meshes. The grid spacing is 2 and the ratio is 1 . The quadrilateral Map meshing method is used for grid that is away from the tennis field. The interval size is 9 and the ratio is 1.01 .

3.2 Boundary conditions. The calculated area size is $100 \mathrm{~cm} \times 100 \mathrm{~cm}$, which is larger than the size oftennis. The upper and lower boundary of the calculation area is set as a symmetric boundary without backflow. The outflow boundary condition is adopted as an outlet boundary, while the velocity-inlet boundary condition is appliedas an inlet boundary. The rotating wall boundary is used for the wall condition.

3.3 Model selection.The diameter of the ball used in the tennis tournament is $6.35 \mathrm{~cm}-6.67 \mathrm{~cm}$. In the simulation, the diameter of the spherical model is set as $6.50 \mathrm{~cm}$. Aim of the simulation is to investigate the flow field and aerodynamic characteristics of the tennis in the windward flying process. In this paper, the concept of relative velocity is used. The tennis is set as the reference system (stationary in the flying direction with rotating), the air is introduced in the way of relative velocity to simplify the simulation model and process. In this study, the flight speed and rotational velocityare directly substituted for the simulation of the ball hitting process. The temperature, pressure and wind speed of air field that the tennisfly inare assumed to be constant. The roughness ofball surface is also assumed to be constant.The front wind of tennis is considered and the impact of lateral wind is ignored.These three forces of gravity, resistance and Magnus force are added to the momentum conservation equation of the ball flight process.

The variation of the surrounding flow field is complex in the flight process of tennis, so the Realizable k- $\varepsilon$ model that has strong adaptability is used and the near-wall region is processed by the wall function method.The mathematical physics expression of the turbulence model is as 
follows:

$\frac{\partial(\rho \kappa)}{\partial \mathrm{t}}+\frac{\partial\left(\rho \kappa u_{i}\right)}{\partial \mathrm{x}_{\mathrm{i}}}=\frac{\partial}{\partial \mathrm{x}_{\mathrm{j}}}\left[\left(\mu+\frac{\mu_{\mathrm{t}}}{\sigma_{\kappa}}\right) \frac{\partial \kappa}{\partial \mathrm{x}_{\mathrm{j}}}\right]+\mathrm{G}_{\kappa}-\rho \varepsilon$

$\frac{\partial(\rho \varepsilon)}{\partial \mathrm{t}}+\frac{\partial\left(\rho \varepsilon \mathrm{u}_{\mathrm{i}}\right)}{\partial \mathrm{x}_{\mathrm{i}}}=\frac{\partial}{\partial \mathrm{x}_{\mathrm{j}}}\left[\left(\mu+\frac{\mu_{\mathrm{t}}}{\sigma_{\varepsilon}}\right) \frac{\partial \varepsilon}{\partial \mathrm{x}_{\mathrm{j}}}\right]+\rho \mathrm{C}_{1} \mathrm{E} \varepsilon-\rho \mathrm{C}_{2} \frac{\varepsilon^{2}}{\kappa+\sqrt{v \varepsilon}}$

Where $_{\varepsilon}=1.2, \sigma_{\kappa}=1.0, \mathrm{C}_{2}=1.9, \mathrm{C}_{1}=\max \left(0.43, \frac{\eta}{\eta+5}\right), \eta=\left(2 \mathrm{E}_{\mathrm{ij}}, \mathrm{E}_{\mathrm{ij}}\right)^{1 / 2} \frac{\kappa}{\varepsilon}, \mathrm{E}_{\mathrm{ij}}=\frac{1}{2}\left(\frac{\partial \mathrm{u}_{\mathrm{i}}}{\partial \mathrm{x}_{\mathrm{j}}}+\frac{\partial \mathrm{u}_{\mathrm{j}}}{\partial \mathrm{x}_{\mathrm{i}}}\right)$

\section{Results and analysis}

4.1The response of characteristics of airflow filed to the kinstate of tennis.In order to analyze the characteristics of airflow field around the tennis in different motion states, three representative conditions with the speed $20 \mathrm{~m} / \mathrm{s}$ and rotationalvelocity $40 \mathrm{rad} / \mathrm{s}$, the speed $20 \mathrm{~m} / \mathrm{s}$ and rotationalvelocity $60 \mathrm{rad} / \mathrm{s}$, the speed $40 \mathrm{~m} / \mathrm{s}$ and rotationalvelocity 40rad/sare selected. Pressure and velocity vectors of the flow field around the ball in each simulation condition are shown in Fig.1.
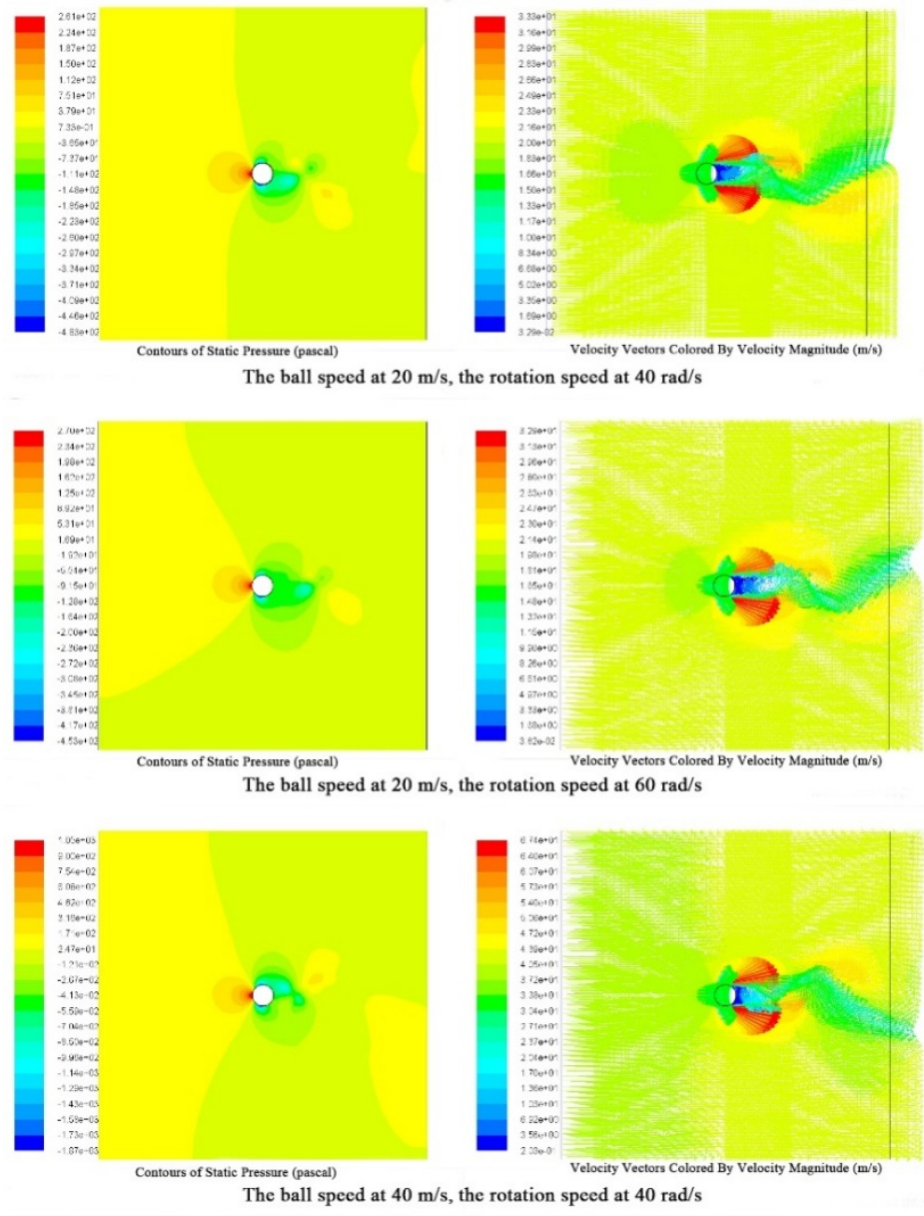

Fig.1. Contours of pressure and velocity

Fig.1shows thatfor thelow velocity, the rotationalvelocityof tennis has little effect on itssurrounding airflow rate, but the pressure distribution around it changes significantly. When the rotational velocity increases, the pressure on the lower surface decreases.It means thedifference in pressure between the upper and lower surface increases, and the separation location of the boundary layer movestothe wind side of tennis. Magnus effect is more obvious with the increase of rotationalvelocity, which results in the greater falling forceand the fast drop of the ball.The change of airflow field and velocity vector around the sphere caused by the change of tennis velocity are also shown in Fig.1. When the tennis speed increases, the velocity of airflow around the ball increases greatly, and the wake flow flexesdownward. As Reynolds number increases with the increases of tennis speed, air flow around tennis becomes disorder. The separation position of boundary layer and the resistance coefficient also change. However, the resistance of flying tennis 
increases, which makes the deteriorated controllability of movement and obvious changes of trajectory.

4.2Distribution of static pressure on the upper and lower surfaces of tennis. The Magnus effect of rotational tennis is influenced by pressure on the upper and lower surface. In this section, rotational speed of $40 \mathrm{rad} / \mathrm{s}$ and the speed of $30 \mathrm{~m} / \mathrm{s}$ are taken as an example to analyze the distribution of static pressure on the upper and lower surfaces.The results are shown in Fig.2.

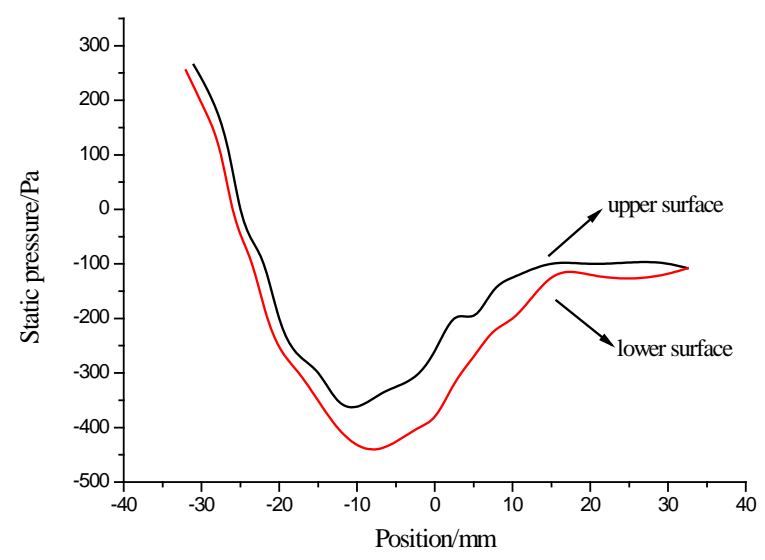

Fig.2. The top and bottom surface of the static pressure distribution

For the upper and lower surface, the distribution law of static pressure is similar, which decreases first and then increases along the direction of horizontal axis, However, the pressure of lower surface changes slightly if compared withupper surface.The reason for the similar distribution is that the airflow field and the surface condition are similar. In the latter part of tennis, the attachment surface layers separate gradually, and leads to the formation of vacuum in local andthe decrease of static pressure.Because of the small velocity of rear air, the static pressure increases gradually. Compared with the upper surface, the airflow velocity of the lower surface is high and the thickness of the surface layer is also larger. Difference in the thickness of surface layers causes various distribution of the static pressure. The static pressure of the ball on the surface is bigger than that in the corresponding location of the surface, so the process of rotation will be subject to a falling force (Magnus Effect). This effect produces the lager falling speed, higher bounce and largerforward momentum.

4.3 Analysis of aerodynamics parameters. The aerodynamic parameters, namelythe drag coefficient and lift coefficient, are obtained from the numerical simulation tests under variousrotational and flight speeds. The physical expressions of drag coefficient and lift coefficient areEq. 3 and Eq. 4,respectively. The aerodynamic parameters represent the relationship between the resistance (the Magnus force) and the relative airflow speed. It is helpful to analyze the characteristics and regulation of force in the flight processof tennis. The drag coefficient is related to the $\operatorname{Re}($ Reynolds), and the lift coefficient is related to the rotational rate $\alpha$. The expressions of $\operatorname{Re}$ and $\alpha$ aretheEq. 5 andEq.6.

$$
\begin{aligned}
& \mathrm{C}_{\mathrm{D}}=\frac{\mathrm{F}_{\mathrm{D}}}{\frac{1}{2} \rho \mathrm{V}^{2} \mathrm{~A}}=\mathrm{f}\left(\mathrm{R}_{\mathrm{e}}\right) \\
& \mathrm{C}_{\mathrm{L}}=\frac{\mathrm{F}_{\mathrm{L}}}{\frac{1}{2} \rho \mathrm{V}^{2} \mathrm{~A}}=\mathrm{f}(\alpha) \\
& \mathrm{R}_{\mathrm{e}}=\frac{\rho \mathrm{Vd}}{\mu} \\
& \alpha=\frac{1}{2} \frac{\mathrm{\omega d}}{\mathrm{V}}
\end{aligned}
$$

Where $C_{D}$ and $C_{L}$ are dimensionless parameters, $F_{D}$ and $F_{L}$ are air resistance and Magnus force, $\rho$ isair density, $\mathrm{V}$ isrelative airflow speed, $\mathrm{A}$ is the windward cross-sectional area oftennis, dis the tennis diameter, $\mu$ is the dynamic viscosity of air, $\omega$ is the rotational velocity.

The aerodynamic parameters in each simulation test are shown in Table 1 . The changes of the drag coefficient and the lift coefficient with the speed and rotational speed as shown in Fig.3to Fig.6. 


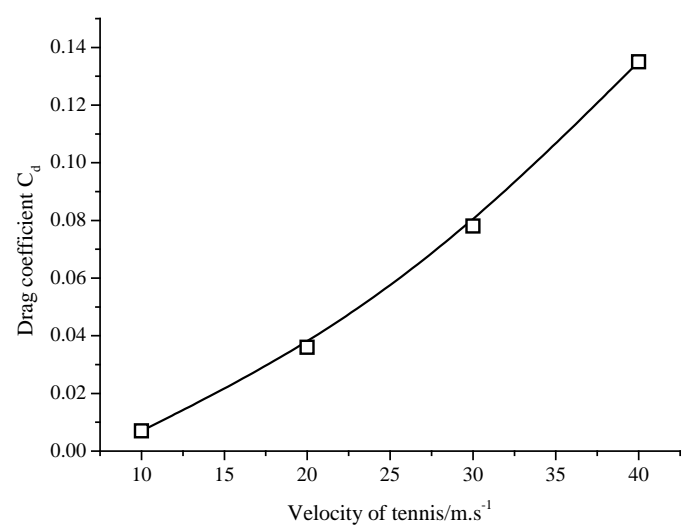

Fig.3. The relationship of Resistance coefficient and sphere velocity

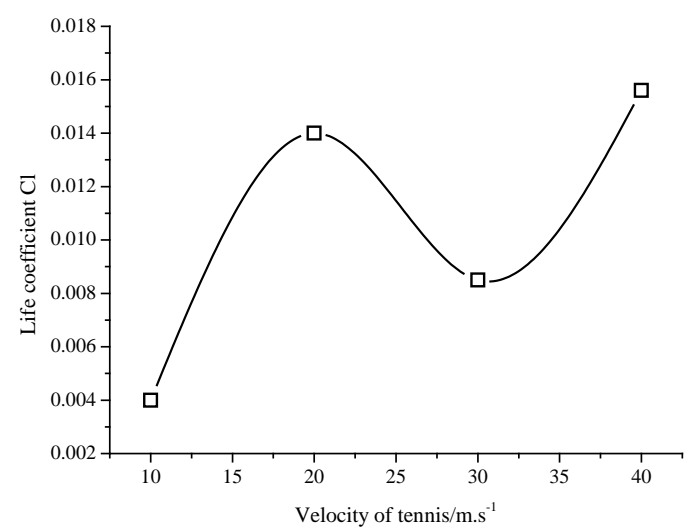

Fig.5. The relationship of lift coefficient and spherical velocity

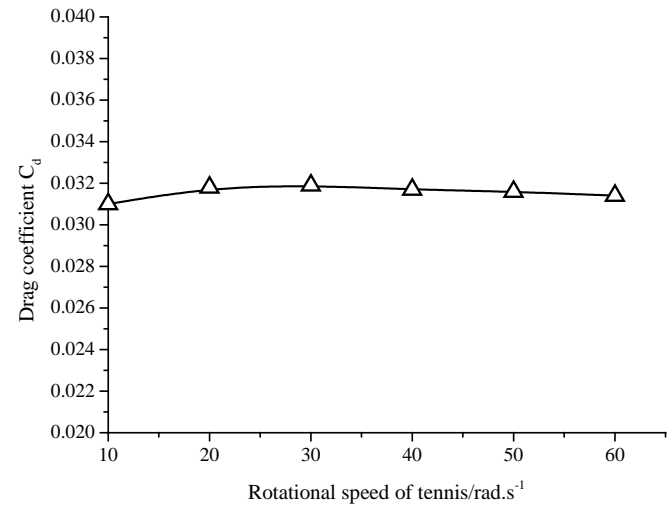

Fig.4. The relationship of drag coefficient and spherical rotation velocity

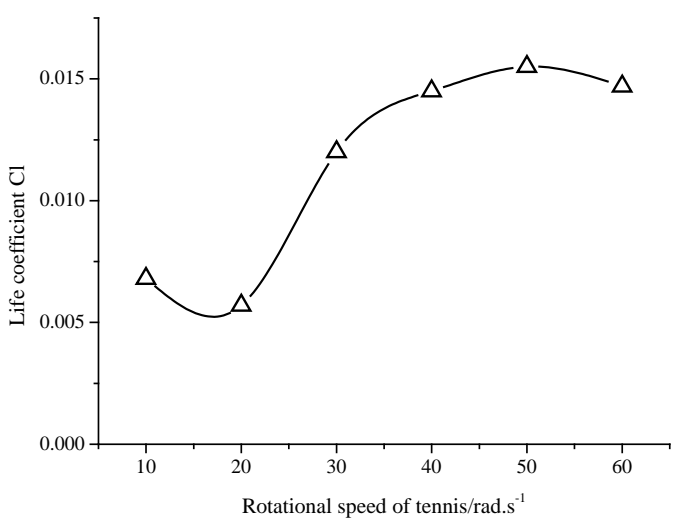

Fig.6. The relationship of the relationship of lift coefficient and spherical rotation velocity

Table 1. CFD simulation results of the aerodynamic parameters

\begin{tabular}{ccccc}
\hline Test number & $\begin{array}{c}\text { Tennis rotational } \\
\text { speed [rad/s] }\end{array}$ & Ball speed $[\mathrm{m} / \mathrm{s}]$ & $\begin{array}{c}\text { Resistance } \\
\text { Coefficient Cd }\end{array}$ & $\begin{array}{c}\text { Lift } \\
\text { coefficientCl }\end{array}$ \\
\hline 1 & 40 & 10 & 0.007 & 0.004 \\
2 & 40 & 20 & 0.032 & 0.014 \\
3 & 40 & 30 & 0.076 & 0.008 \\
4 & 40 & 40 & 0.133 & 0.015 \\
5 & 10 & 20 & 0.031 & 0.007 \\
6 & 20 & 20 & 0.032 & 0.006 \\
7 & 30 & 20 & 0.032 & 0.012 \\
8 & 40 & 20 & 0.032 & 0.014 \\
9 & 50 & 20 & 0.032 & 0.016 \\
10 & 60 & 20 & 0.031 & 0.014 \\
\hline
\end{tabular}

The simulation result shows that the drag coefficient increases with the tennis speed for a certain Reynolds number, while the lift coefficient fluctuates greatly and the regularity is not obvious. When the rotational speed increases, the difference in pressure between upper and lower surface shows a change. The lift coefficient presents an increasing trend, and the resistance coefficient nearlydo not change. The resultdemonstrates that tennis velocity has a great influence on the drag coefficient, and the rotational speed mainly affects the lift coefficient. Firoz, Alam, etc. have conducted the changes oftennis aerodynamic parameters with the flight and rotational speedsusing the wind tunnel test [8]. The test results show thatthe drag coefficient increases first 
then decreases slightly with a certain increase of tennis speed, but the change of lift coefficient is not obvious. With the increase of rotational speed, the lift coefficient increases and the drag coefficient fluctuates greatly. The main reason for the difference between the simulation test and the wind tunnel test is that the surface roughness of the tennis is assumed to be constant in the simulation, while the presence of fluffs causes the roughness of the tennis surface changing with the wind speed in the wind tunnel test. Under the condition of low flying speed, the fluffs make the increase of spherical roughness, but the 'smooth' surface will form if the tennis speed reaches a certain value because of the close combination between the fluffs and tennis.The change of roughness greatly influences the change rules of drag coefficient and lift coefficient with the motion parameter of the tennis. In addition, the motion of the tennis is affected by the combined influence of the frontal wind and the lateral wind in the wind tunnel test. In this paper, only the frontal wind is included in the simulation experiment.

\section{Conclusion}

In the flying process of tennis, the characteristics of airflow field around tennis and the aerodynamic parameters are influenced by its motorial conditions. Based on the analysis offorce factors in the motion process, the momentum conservation equation of simulation experiment is confirmed by considering gravity, resistance and Magnus force. A multidimensional simulation test are conducted by simplifying the movement of the tennis to be static but rotation. The main conclusions of this paper are shown as follows.

1.The rotational velocity oftennis has little effect on the ambient air velocity, but it affects the pressure distribution obviously. For a largetennis speed, the difference in pressure between the upper and lower surfacesand the Magnus effect increase, which result in the increaseof 'falling force'. The increase of tennis velocitynot only causes a significant increase of speed for the surrounding airflow, but also leads to the disorder of air flow, poor controllability and trajectory change of tennis.

2.The airflow field of the upper and lower surfaces ofrotational tennis is similar, so the distribution of static pressure is also nearly the same. However, the pressure of lower surface changes slightly behind that of the upper surface and the static pressure of the lower surface is less than that of the corresponding position of upper surface because ofhigh air velocity and thickness of lower surface. 3.Computationresults show thatthe drag coefficient increases with the tennis speed, and the lift coefficient fluctuates greatly without regularity. With the increase of rotational speed, the lift coefficient increases, but the drag coefficient keeps constant. In the simulation test, the roughness of the tennissurface is assumed to be constant. This results in the different relations between the resistance coefficient and rotational speed. On the other hand, the relationship between lift coefficient and tennis velocity is also different from that in the wind tunnel test.The subsequent simulation test will optimize the surface roughness setting of different balls, and improve the resultsof numerical simulation.

\section{Literature References}

[1] A. J. Cooke: Sports Eng Vol. 3(2000), p. 123-129

[2] A. Nakashima, T. Okamoto and Y. Hayakawa: IFAC Proceedings Volumes Vol. 47(2014), p. 7176-7181

[3] R. Cross and C. Lindsey: Sports Eng Vol. 17(2014), p. 89-96

[4] R. Cross and C. Lindsey: Eur. J. Phys Vol. 38(2017), p. 044002

[5] R. Mehta, F. Alam and A. Subic: Sports Technol Vol. 1(2008), p. 7-16

[6] S. Barber and M. J. Carré: Sports Eng Vol. 13(2010), p. 47-55

[7] S. R. Goodwill, S. B. Chin and S. J. Haake: J. Wind Eng. Ind. Aerod Vol. 92(2004), p. 935-958

[8] F. Alam, A.J. Subic, J. Naser, M.G.Rasul and M.M.K. Khan:WseasTransac on Fluid Mecha Vol. 3(2008), p. 271-278. 